CARNETS OE Carnets de géographes

GÉOGRAPHES.

4 | 2012

Géographies critiques

\title{
Géographie de la littéracie
}

close et distant reading au Mali

Frédéric Barbe

\section{OpenEdition}

Journals

Édition électronique

URL : http://journals.openedition.org/cdg/985

DOI : $10.4000 / c d g .985$

ISSN : 2107-7266

Éditeur

UMR 245 - CESSMA

Référence électronique

Frédéric Barbe, « Géographie de la littéracie », Carnets de géographes [En ligne], 4 | 2012, mis en ligne le 01 septembre 2012, consulté le 04 mai 2019. URL : http://journals.openedition.org/cdg/985 ; DOI :

10.4000/cdg.985

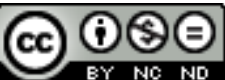

La revue Carnets de géographes est mise à disposition selon les termes de la Licence Creative Commons Attribution - Pas d'Utilisation Commerciale - Pas de Modification 4.0 International. 


\title{
GEOGRAPHIE DE LA LITTERACIE
}

Close et distant reading au Mali

FREDERIC BARBE

Doctorant en géographie à I'Université de Nantes

UMR 6590 ESO

frederic.barbe@univ-nantes.fr

\section{Résumé}

Issu de l'anglais literacy, francisé au Québec en 2002, le terme de littéracie désigne ici, au sens large, les acteurs, les usages, les objets et les politiques de lecture-écriture, à travers le monde. "Pratiquer" en géographe (ou en militant) la littéracie, c'est accepter de s'éloigner du registre simple de la grande culture et de la croyance littéraire pour se saisir d'une matière fabuleuse, un monstre de la mondialisation, la "bibliothèque mondiale ", aux ressources infinies à notre échelle de perception. C'est jouer des échelles, entre l'œuvre et la série, le proche et le lointain. C'est aussi étendre les mesures de la valeur, à l'informel, aux langues dominées (de l'international au domestique), aux formes de la marge, loin du "grand partage " primitif/civilisé qui traîne encore dans beaucoup d'imaginaires. Une enquête en littéracie malienne (2011) suggère à la fois la profonde normalité du système culturel malien et la force du système transcolonial qui a fait échouer l'utopie scolaire de l'Indépendance. Une géographie critique appliquée à la culture permet ici de repérer, contre un certain nombre de mythologies agissantes, des obstacles à la formation d'un marché national de la littéracie cohérent et de suggérer une action publique plus soucieuse de ménager le territoire et la société en favorisant un accès universel à la lecture-écriture au Mali.

\begin{abstract}
Littéracie, from the English word "literacy " (gallicized in Quebec back in 2002), designates in its widest sense the reading-and-writing actors, usages, objects and policies from all over the world. Practicing literacy as a geographer or even militating for it means accepting to distance oneself from the simple register of grande culture as a whole and from literary faith in order to take over a fabulous subject, a globalization monste, the "world library ", and its endless resources (from our scale of perception). It means using various scales, alternating from single work to mass-production and edition, from the close to the distant. It also implies widening the value measures to an informal extent, to the widely-spread languages (from the international to the domestic aspect), to the margin form, far away from the huge primitive/civilized
\end{abstract}


division which still lies in many people's mind. A 2011 study on Malian literacy means to suggest that both the great normality of the Malian cultural system and the power of the Malian transcolonial system contributed to the failure of Independence scholar utopia. A critical geography, when applied to culture, and in spite of active mythologies, enables to spot the obstacles to the formation of a coherent literacy national market and to recommend that public action be taken in a more aware way in terms of territory and society, favoring at the same time a universal access to the whole reading-and-writing subject in Mali.

Le terme de littéracie, tel qu'entendu dans cet article par un géographe, désigne les acteurs, les usages, les objets et les politiques de lecture-écriture à travers le monde. Dans la culture anglo-saxonne, le terme vulgaire de literacy a acquis un sens politique et scientifique à la fin du $19^{\text {ème }}$ siècle, permettant au cours du siècle suivant, le développement d'un vaste champ de recherche pluridisciplinaire, doté de nombreux terrains, au Nord comme au Sud et sur la relation même Nord-Sud, marqué de figures significatives (Jack Goody, Richard Hoggart, Edward Saïd, par exemple). Ce courant de recherche se déploie aujourd'hui dans les New Literacy Studies. Un ouvrage comme Africa's hidden histories : everyday literacy and making the self, dirigé par Karin Barber (2006) rend compte de la richesse de ce mouvement. Dans notre contexte national qui méconnaît largement le concept et l'a emprunté aux Québecois ${ }^{1}$, " pratiquer " en géographe (ou en militant) la littéracie, c'est d'abord s'obliger à déconstruire une croyance littéraire fortement indurée dans la grande culture française. Cette croyance littéraire tend à réduire le champ légitime de l'écriture et de la lecture à la "littérature " et plus encore à son "canon", soit une part infime du corpus écrit disponible. En cultivant le mythe de l'extra-territorialité de l'écrivain et l'irréductibilité de l'œuvre, en défendant, contre toute science sociale, une figure étroite du don, la croyance littéraire évacue l'écriture et la lecture du registre socio-spatial.

\section{Une matière fabuleuse, un monde de l'art}

Le géographe conséquent ne peut se satisfaire de travailler sur les seules représentations géographiques dans les œuvres du canon littéraire, segment aujourd'hui bien documenté. Si la géographie critique a quelque chose à voir avec la littéracie, c'est d'abord dans une revendication d'accès à un énorme corpus écrit, qu'il

\footnotetext{
1 Selon l'Office québecois de la langue française, qui fait entrer le terme dans son dictionnaire en 2002, la littératie (n.f.) est l'ensemble des connaissances en lecture et en écriture permettant à une personne d'être fonctionnelle en société. Il existe une variante graphique : littéracie.

Note : le seuil de connaissances nécessaires pour être fonctionnel change au fil du temps et est variable d'une société à l'autre. L'ensemble des connaissances acquises doit permettre à une personne de lire et de comprendre des textes de trois types : des textes suivis (articles de journaux), des textes schématiques (cartes routières) et des textes à contenu quantitatif (calcul de l'intérêt sur un emprunt). La mesure du niveau de littératie fournit un indicateur économique; plus le niveau de littératie d'une personne est élevé, meilleures sont ses chances d'occuper un emploi rémunérateur. En français, la finale -tie, prononcée "si », est plus fréquente que la finale -cie.

J'ai choisi volontairement dans cet article de conserver la variante graphique que j'avais initialement utilisée en début de recherche, "littéracie ", qui, dans ma propre subjectivité, m’apparaît plus vernaculaire et plastique.
} 
faut bien nommer la "bibliothèque mondiale ", mais aussi à une quantité incroyable d'acteurs, d'usages et de politiques de lecture-écriture. Dans cet objet extravagant, dans cette matière fabuleuse, il y a bien plus qu'un simple champ professionnel aux acteurs légitimes dotés de capitaux spécifiques et de positions. Nous y voyons, à la manière d'Howard Becker, un véritable "monde de l'art", que de nombreux " inventeurs » français ont exploré depuis leur propre lieu (Roger Chartier, Georges Perec, Pierre Bayard, Pascale Casanova, Bernard Lahire, Jacques Rancière, Daniel Fabre, Jean Hébrard, Gisèle Sapiro, Daniele Morante, Aïssatou Mbodj-Pouye, etc.) aux côtés des habitants ordinaires. Cette extension du corpus fonctionne comme une véritable entreprise scalaire. Franco Moretti, chercheur en littérature passé à la géographie, a montré combien était fructueux ce jeu scalaire permanent entre le close reading (l'échelle de l'œuvre, sinon du fragment) et le distant reading (l'échelle de séries d'objets à construire, à inventer).

\section{Sortie de l'aporie transcoloniale, la littéracie malienne}

Engagé dans un travail doctoral sur plusieurs terrains d'échelle différente, nous exposons ici quelques éléments d'une recherche menée sur un espace national au Sud, la République du Mali. Au delà des clichés néo-hégéliens, nous croyons observer un mode d'émergence "sous contraintes et à toute vitesse", qui nous dit à la fois la normalité de la littéracie malienne et ses profondes difficultés à atteindre les normes et les objectifs d'une société pleinement alphabétisée. Dans ce contexte exigeant de la pauvreté matérielle et de la très forte extraversion, les politiques culturelles du jeune État malien sont indéniablement une géographie appliquée et autoréférente : le Mali fabrique du Mali, sous l'œil discret mais ferme des partenaires techniques et financiers. Les ratés de cette fabrication nationale, notamment un certain nombre d'apories dans la littéracie locale, obligent le chercheur qui s'est rapproché d'une position d'observation participante à assumer des positions scientifiques parfois iconoclastes. Ici, les politiques d'ajustement structurel, l'idéologie du tout marché, mais aussi l'utopie francophone, l'illusion d'un modèle français de l'édition et de la librairie universel, ou bien encore l'abus de position dominante des grands éditeurs français sur le marché malien posent à l'évidence des questions à la société et à la politique. Au Mali, en Afrique de l'Ouest, mais aussi en France ou dans les organisations internationales. La géographie de la littéracie permet ainsi de revisiter d'une manière heuristique quelques problématiques de la mondialisation contemporaine, telle l'entrée transcoloniale, saisissante au Mali. La littéracie se développe bien comme une relation spatialisée.

\section{La littéracie, aux frontières de la géographie}

Nous parlons ici d'une frontière dans la géographie entre le légitime et le non-légitime. À la fois certaine et malaisée à voir sur le terrain, cette frontière épistémologique nous tient à l'écart de certains acteurs, de certains objets, de certaines approches, à différentes échelles et pour différentes raisons. Ce sont les frontières de l'archipel de la géographie, un territoire partagé entre pratiques légitimes et non-légitimes dans la corporation. Sur une telle frontière, le passage en littéracie offre une formidable opportunité de trabendo scientifique, où le gain s'appelle échelle, corpus et éthique. À l'évidence, la révolution numérique multiplie les enjeux dans les trois domaines. On 
entendra, en bon trabendiste, que la littéracie est un projet vivant et plastique, qui se déploie de l'oralité classique au multimédia le plus contemporain.

\section{Le jeu scalaire en littéracie}

Appliquer l'échelle au texte écrit. Le plan n'est pas neuf et les émergences nationales l'ont utilisé dans les constructions stato-nationales: promotion des langues vulgaires (nationales) face au latin à l'époque moderne ou, plus tard, face à d'autres langues nationales concurrentes (futures langues régionales) dans le développement de l'institution scolaire et d'un corpus aux dimensions du marché national. Au même moment, une nouvelle littéracie coloniale génère des phénomènes exacerbés de concurrence entre langues, corpus, usages et acteurs dans les sociétés assujetties et entretient le mythe d'un monde non-occidental pré-moderne frappé d'oralité (pas de langue écrite, pas de corpus, pas d'histoire) et de babélisme (une fragmentation extrême du champ linguistique proche de l'incommunicabilité). Ce " grand partage " entre civilisé et primitif dans les deux échelles spatiale et temporelle a été profondément remis en cause. Jack Goody (1977) a montré que la raison graphique (le texte et les images de pensée - croquis, dessins, cartes, etc.) n'oppose pas deux catégories de sociétés, mais est un processus continu qui signe l'ensemble des sociétés humaines et se développe en fonction de nombreuses interactions sociales et spatiales. Plus récemment, Daniele Morante (2009) déconstruit le babélisme, représentation erronée du réel, qui surestime la diversité linguistique au Sud et la sous-estime au Nord. En expliquant la diversité des langues dans le monde par un "système gravitationnel linguistique ", le socio-linguiste montre que la distance (les distances métrique, économique, politique, culturelle, juridique) est le principal facteur d'évolution des langues.

Appliquer l'échelle au texte écrit, c'est aussi inventer de nouvelles manières d'investiger. Franco Moretti, s'il ne s'échappe que marginalement de la littérature, l'entend large, systématique (tous les genres, au delà du canon) et ouverte à I'imaginaire du chercheur. II écrit ainsi dans Graphes, cartes et arbres, modèles abstraits pour une autre histoire de la littérature (2005, 2008 pour l'édition française), [qu'ici] la réalité du texte subit un processus de réduction et d'abstraction délibérée. "Lecture de loin" (distant reading), ainsi ai-je nommé ce type d'approche il y a quelques années - où la distance n'est pas un obstacle, mais une forme spécifique de connaissance: un nombre plus réduit d'éléments, d'où un sens plus aigu de leur interconnexion globale. Organisations, relations, structures. Formes. Modèles. II renouvelle une lecture darwinienne de la littérature : divergence, convergence, cycles, niches, diversité. Dans une recherche (parution en cours) sur la littéracie des îles Aran, haut-lieu de la renaissance culturelle irlandaise à partir des années 1880, archipel minuscule et démographiquement déclinant du Gaeltacht à l'entrée de la baie de Galway, nous avons pu montrer que non seulement les auteurs (John Synge, Robert Flaherty et John Messenger pour les plus significatifs) viennent sur l'archipel pour y occuper ou y créer des niches très spécifiques, mais qu'ils utilisent également les trois îles de l'archipel dans un sorte de spécialisation liant une posture à une île : Inishmore pour la forme touristique, Inishmaan pour la forme indigéniste et Inisheer pour la déconstruction des deux formes précédentes. Le développement de la littéracie indigène (usage du bilinguisme, convergence du genre, accès aux nouveaux médias, migrations, tourisme) lié à une intégration de plus en plus poussée (et donc une 
réduction des distances) permet l'émergence d'auteurs et d'acteurs culturels insulaires de grande qualité comme Pat Mullen, local hero singulier ou Liam O'Flaherty et son génial Tourist's Guide to Ireland (1929). Le terrain irlandais se présente ainsi comme un exemple européen de mutation d'une littéracie colonisée, suggérant des transferts intéressants vers d'autres parties du monde.

\section{La bibliothèque mondiale, un monstre}

Dans notre acception, l'expression désigne l'ensemble du corpus écrit mondial effectivement disponible. Tout à la fois objet physique à la forte matérialité et représentation intellectuelle complexe, la bibliothèque mondiale (ou n'importe qu'elle autre) nous semble un espace géographique de plein exercice. Cette bibliothèque " intensive" historiquement inscrite dans le long processus d'accroissement des productions écrites ne se différencie guère, au premier abord, de la bibliothèque " extensive " de tel ou telle d'entre nous - et qui ne nous survivra pas longtemps. Pour construire les interactions entre toutes ces (échelles de) bibliothèques, il faut les passer dans les nouvelles humanités proposées à nos étudiants. "Spatialiser sa bibliothèque " en utilisant les ressources de la discipline est une procédure réflexive particulièrement riche. La spatialisation de sa bibliothèque peut être facilement collectivisée pour devenir la bibliothèque partagée des étudiants de géographie et acquérir un véritable statut épistémologique et professionnel (découvrir les images de pensée, orienter les lectures, construire un projet pour sa bibliothèque personnelle, voire, pour les plus audacieux y travailler un projet d'écriture personnel). En faisant cette recherche (Barbe, 2011), nous constatons avec les étudiants que les bibliothèques relèvent d'une véritable morphogénèse culturelle (transport, dépôt, érosion), travaillant les catégories de la bibliomasse et de la bibliodiversité.

Dans cet ensemble, la bibliothèque mondiale est un monstre à part : catalogues, livres, littératures grises, inventaires, archives publiques ou privées, pièces comptables, procès-verbaux, fichiers électroniques, data base, puces, deep ou hidden web, carnets, cahiers, graffitis, tatouages, affiches, dessins, etc. empruntant toutes sortes de formes matérielles et de statuts. Travailler la question de la bibliothèque mondiale, c'est donc s'inscrire soi et inscrire la bibliothèque elle-même dans une profonde hétérogénéité, celle du grand nombre de ses individus, de ses propriétés et de ses innombrables combinaisons de formes et d'échelles. La croissance exponentielle du nombre de livres disponibles que Pierre Bayard a magistralement mise en scène dans Comment parler des livres que l'on n'a pas lus (2007) est l'un des devenirs de cette bibliothèque mondiale. Face à l'explosion des lectures possibles, le lecteur doit construire de nouvelles stratégies. Dans le monde de l'art de la littéracie, la reconnaissance du lecteur émancipé, de la légitimité du bricolage, du marronnage cher à Kossi Efoui ${ }^{2}$, des écritures ordinaires, mais aussi de l'oubli, du désordre créateur, de la difficulté à constituer le canon deviennent des idées nécessaires. Dans le même temps, les métiers traditionnellement associées à l'écrit (enseignants, écrivains et éditeurs) entrent (et "sont entrés" par force) dans une logique néo-libérale qui intensifie les phénomènes de concurrence pour l'accès au lecteur ordinaire. Le "modèle de l'oligopole à franges " montre qu'au delà du cœur de marché contrôlé par quelques 
groupes transnationaux, une part significative de l'invention lettrée vient des structures petites et moyennes, dont s'inspirent les premiers. La faillite, le rebut ou pilon y régulent les errances du marché. En dématérialisant partiellement la bibliothèque, la révolution numérique amplifie les enjeux, du côté de la propriété intellectuelle comme de la protection de la vie privée. Les écritures automatisées dérivent des réseaux sociaux archivant de manière fantastique la moindre donnée des utilisateurs et des projets " d'internet des objets " géolocalisant et analysant dans d'immenses bases de données des millions d'objets en temps réel. Pour autant, le livre papier et les écritures ordinaires (Fabre, 1993, Mbodj-Pouye, 2007) comme les cahiers, carnets et brouillons personnels semblent ancrés dans toutes les sociétés, même les plus pauvres. Devant ces mutations, le discours de la croyance littéraire se fait souvent agressif et réactionnaire dans une dénonciation paradoxale de l'enrichissement technologique et culturel. L'humanisme à la Frédéric Dard nous parle alors avec bonheur de cette extension inattendue de la géographie. II y a vingt-cinq ans, je suis allé, avec ma femme Françoise, en Afrique. Je me trouvais, pour une escale, à l'aéroport de Niamey. Dans le hall, il y avait un type qui vendait, sur une natte posée à même le sol, des morceaux de canne à sucre et des "San-Antonio " jaunis. C'est l'image qui, tout au long de ma carrière, m'a le plus frappé, le plus ému. J'ai compris ce jour-là que mes bouquins, ça n'était pas de la littérature, c'était une denrée. Une denrée comestible, bon marché, et universelle ${ }^{3}$.

\section{L'épuisement du grand partage colonial}

Montrez-moi le Tolstoï zoulou ! Cette répartie d'un intellectuel nord-américain est rapportée par Edward Saïd dans Culture et impérialisme (1993, 2000 pour la traduction française). L'universitaire américano-palestinien y reconnaissait alors les propos de ceux qui, aujourd'hui, parlent de l'Occident et de ce qu'il a fait et sur ce que le reste du monde a été, est et peut être 4 . Nous pourrions dire également que l'injonction paradoxale de son contradicteur illustre habilement la croyance littéraire en la transposant dans l'espace mondial. Pour explorer le déplacement ou la mutation des instances internationales de légitimation littéraire, nous avons proposé une Tentative d'épuisement du et des prix Nobel (Barbe, 2011). L'étude des ressources spatiales mobilisées par les 105 écrivains nobelisés montre que la centralité occidentale, secondairement française, germanique, nordique et nord-américaine, du prix Nobel de littérature n'empêche pas la reconnaissance progressive de grands auteurs du Sud et de l'Est. II apparaît même que beaucoup de titulaires du Nobel de littérature, quelle que soit leur origine, partagent sinon une appétence, du moins une même expérience de la mobilité (expatriation, exil, retour au pays, résidence, nomadisme, brain drain, brain drain reverse, brain circulation). Au fond, l'institution Nobel résout discrètement la question des inégalités dans le champ littéraire dans le monde par la valorisation de créateurs qui, à un certain moment de leur carrière, ne sont plus du Sud, du Nord ou de l'Est, mais vivent avant tout dans le segment le plus mondialisé du monde de l'art de la littéracie. Les modèles de formation des marchés littéraires nationaux et de constitution de lieux de légitimation décrits par Pascale Casanova (1999) permettent de documenter ces passages, où art, culture, politique et moyens de subsistance se 
mélangent. On voit de suite que l'hypothèse d'une normalité malienne se nourrit aussi de ces jeux d'échelles, comme d'un nécessaire travail de terrain. En effet, trop de textes sur la littéracie africaine affichent des données dramatiquement dérisoires et fausses. La réalité des publications au Mali (ou en RDC, étudiée par Cassiau-Haurie, 2007), si elle est difficile à saisir, montre à la fois une production locale supérieure aux données nationales ou internationales et une crise de la littéracie africaine à partir des années 1980 : ni les pratiques ordinaires liées à la scolarisation, ni la constitution de jeunes littératures nationales ne résistent vraiment à la crise de la dette et de la souveraineté, à la décomposition des nouveaux États-nations. Les politiques d'ajustement structurel et la marchandisation du fait social qui l'accompagnent ont eu et continuent d'avoir des effets sur la littéracie au Sud. Nous croyons voir là un ultime effet de la croyance littéraire, que d'imaginer que la littéracie ne puisse être atteinte par la destruction des services publics et de l'économie formelle d'un pays

On voit alors que le "grand partage " primitif /civilisé chassé par la porte scientifique (Goodis, les New Literacy Studies) peut revenir par la fenêtre politico-médiatique d'une vulgate à base de "négrologie" (Smith, 2004) ou d'enthousiasme néo-hégelien (Guaino, 2007). Ainsi le Malien n'aimerait pas lire ou préfèrerait monnayer les notes de ses enfants plutôt que de l'éduquer. Ces clichés, locaux ou non, naturalisent des faits sociaux contre l'éthique du réel: une personne qui n'a pas pu accéder à l'alphabétisation peut-elle ne pas aimer lire ? Dans un État nourricier, mais à la fois patrimonialisé, autoritaire et impuissant (à faire appliquer la loi commune comme à répondre au sous-emploi généralisé), l'investissement dans la fraude scolaire se légitime socialement et c'est rester honnête qui devient risqué. Ces processus de naturalisation sont inacceptables et doivent être neutralisés pour qu'un travail scientifique et politique puisse advenir. Les pays émergents, et l'Inde en premier lieu, ont dépassé certaines des contraintes qui enserrent la littéracie du Mali et de bien d'autres pays pauvres. Beaucoup de ces pays partagent une même allure géographique alliant polarisation des revenus et polarisation linguistique : l'élite parle une langue transcoloniale, les classes populaires des langues vernaculaires. Ainsi $45 \%$ des livres édités en Inde le sont en anglais, alors que cette langue n'est maîtrisée que par 3 à $5 \%$ de la population. Mais l'effet de taille permet à l'Inde d'être un gros producteur de livres en langues nationales et le troisième producteur mondial de livres anglophones avec un marché réel de 30 à 50 millions de lecteurs anglophones (Bief, 2005). En changeant de partenaire sans changer de langue transcoloniale (les ÉtatsUnis à la place de l'ancienne puissance coloniale), l'Inde ne s'affranchit pas pour autant d'une position dominée, aux énormes importations de livres occidentaux en langue anglaise (livres neufs et livres neufs soldés dont la filière indienne demande I'interdiction). Le développement des Subaltern Studies se nourrit notamment à cette culture indienne de l'émergence sous domination. Dans Provincializing Europe (2000, 2009 pour la traduction française), Dipesh Chakrabarty réfute la lecture des faits sociohistoriques issue de la téléologie occidentale. Médiateur historique de la société mondiale, porteur unique de la temporalité du progrès et de la modernité, l'Occident selon Chakrabarty repousse et enferme les autres sociétés dans le double registre de l'anachronisme ou de la répétition/imitation. Ce qui est réfuté finalement, c'est une conception du temps historique organisée à partir d'un centre géographique monopolistique et invasif. Béatrice Collignon (2010), en exposant de manière critique le différentiel d'éthique du terrain entre les cultures universitaires française et 
canadienne, ne dit pas autre chose. Incorporation des connaissances traditionnelles " (disons "vernaculaires" pour sortir le propos du seul cadre autochtone) ne doit pas signifier seulement " incorporation des informations " mais bien "incorporation des conceptualisations " autres que celles du savoir scientifique occidental, qui n'est jamais qu'une forme particulière de savoir. Dans cette perspective, le monde de l'art de la littéracie s'occupe bien de produits de haute nécessité (Manifeste de 2009).

\section{Lire-écrire au Mali, une " normalité » avec guillemets}

La recherche menée au Mali en 2011 est doublée d'un compagnonnage de type recherche-action dans la filière du livre. Celui-ci a permis d'aborder les acteurs dans un état d'esprit de type professionnel-égalitaire. Les entretiens menés avec une cinquantaine d'acteurs de l'écrit, formels et informels, l'étude du corpus et des infrastructures, l'approfondissement des questions linguistiques, la découverte via les acteurs d'une situation scolaire sans rapport avec la littérature des PTF, l'envahissement intime par l'ambiance, les représentations, les contradictions, les rêves comme les murs opposés à l'action nous ont amené peu à peu à dresser un état des lieux mélangé et arrangé: nécessité de néologismes, de figures rhétoriques inédites pour dire l'exact de la perception, positionnement prudent et paradoxal, en tant que chercheur français, dans des débats maliens parfois très clivés. La figure de la "normalité " avec guillemets, désigne ici un processus d'émergence paradoxale. Les signes habituels de l'émergence d'une littérature nationale (Le Potvin, 2005) se confrontent dès les années 1970 et 1980 à l'aporie de l'utopie scolaire francophone et à la déflagration née de la crise des ressources et de la souveraineté. Nous allons parler ici de la "langue étrangement nationale " (le français) et de "l'ajustement structurel de la littéracie " (sa déstructuration sous l'effet de la marchandisation imposée).

\section{L'émergence " sous contraintes et à toute vitesse "}

La République du Mali vient de fêter son cinquantenaire (2010). Le Soudan français est créé le 18 août 1890, à partir de la région de Kayes et du Haut Sénégal, point d’entrée de la colonisation française, contrôlant progressivement les autres entités politiques autochtones et installant la capitale administrative, à partir de 1904, dans un ancien centre économique, Bamako. Les limites territoriales sont fixées dans les années cinquante, dix ans seulement avant l'accession à l'indépendance. Ces futures frontières nationales ne recouvrent pas du tout les limites historiques des grands empires ouestafricains et dissimulent des processus de mobilité et d'islamisation toujours à l'œuvre aujourd'hui. Si le temps colonial est marqué par l'arbitraire répressif, la mise en rivalité des groupes ethniques, la sous-intégration (scolaire, sanitaire, logistique), mais aussi par l'exploitation des ressources agricoles (coton, arachide) et le fantasme récurrent du grenier rizicole soudanien (générant notamment le travail forcé et les déplacements de population de l'Office du Niger), on ne peut se contenter d'une description par la coercition et on doit dire avec Achille Mbembé (2010) sa forme massivement idéelle. C'est en partie à cause de sa redoutable capacité de prolifération et de métamorphose qu'il fit trembler le présent de ceux qu'il s'était asservis, s'infiltrant jusque dans leurs songes, remplissant leurs cauchemars les plus affreux, avant de leur arracher d'atroces lamentations.[...] Habituée à vaincre sans avoir raison, [la colonisation] exigea des 
colonisés non seulement qu'ils changent leurs raisons de vivre, mais aussi qu'ils changent de raison - des êtres en écart perpétuel.

De ce point de vue, la République du Mali peut apparaître marquée par une prophétie performative initiale en langue française, sa propre proclamation en 1960 : Le Mali existe, vive la République du Mali ! Une prophétie réitérée aux générations successives d'écoliers, de plus en plus nombreux à réitérer, et qui accouche d'elle-même progressivement: le Mali fabrique du Mali. C'est bien le sens du titre du livre de Moussa Mara "L'État au Mali, Géniteur de la Nation [Facteur d'Intégration, Catalyseur d'expansion] " (2010). Pour approcher cette matière qui choquera les nationalistes coutumiers de la naturalisation des faits sociaux et spatiaux, il faut rencontrer les acteurs eux-mêmes, "remarquables » et " ordinaires ". Inspirée par la geste coloniale (maintien de la langue coloniale, concurrence des deux groupes sociaux les plus proches du colon, enseignants et militaires), cette décolonisation est aussi imbibée (inhibée) de la rivalité des deux modèles, soviétique et occidental. Les outils culturels publics sont immédiatement au cœur du projet unitaire de l'État malien, dans la première période " autoritaire " comme dans la seconde, plus " décentralisatrice ": arts traditionnels (danse, musique, chasse, sculpture), écoles, musées, édition, médias. Il s'agit parfois de modernité classique, le plus souvent de ré-invention de la tradition et de co-construction des récits et des formes (que le "pays dogon " illustre de manière caricaturale; Doquet, 2009). En effet, pour la plupart de ces ressources et productions, nous préférons utiliser le qualificatif de " transcolonial ", signifiant par là qu'elles ne sont ni $a$, ni pré, ni post, mais qu'elles passent par le temps colonial, qu'elles s'y transforment et qu'elles développent la forme contemporaine après la décolonisation. À partir de la figure des " médiateurs coloniaux " (Coquery-Vidrovitch, 1992), nous voyons que la mise en scène de la nation dans la nouvelle modernité malienne n'est pas une rupture, mais plutôt une continuité transcoloniale. Le développement du français au Mali est exemplaire : née de la médiation culturelle coloniale, la langue officielle du Mali est quantitativement déployée par l'État malien indépendant, qui, d'une certaine manière, s'y est enfermé. Modibo Keïta, le premier président du Mali indépendant de 1960 est aussi le gendre du premier interprète officiel de la colonie du Soudan, Moussa Travelé, lui-même premier auteur malien moderne en 1913 (au sens du système industriel édition-imprimerie-librairie), dans une forme elle-même bilingue. L'échec de l'utopie scolaire francophone de Modibo Keïta a montré les limites de la fabrique nationale malienne appuyée sur la langue française, attaquée à la fois par les injonctions libérales de l'ajustement structurel et les errements de la dictature militaire. Le Mali transcolonial a émergé dans une normalité certaine, mais aussi "à toute vitesse et sous contraintes ". La ressource linguistique semble ici toujours particulièrement centrale, voire décisive.

\section{La lutte des langues}

La littéracie malienne se développe dans trois bulles linguistiques enchevêtrées. La bulle francophone, dominante pour la littéracie (livre, presse, net), peut s'apparenter à une forme de rente linguistique, à la fois des catégories les plus aisées et/ou les mieux scolarisées, mais aussi spatialement de la région-capitale ou de segments touristiques

5 Achille Mbembé, Sortir de la grande nuit, essai sur l'Afrique décolonisée, 2010, La Découverte, pages 15 et 16 . 
et migratoires. Bien que concentrant l'essentiel des ressources du champ considéré, la bulle francophone se caractérise par son impossibilité à croître de manière significative aujourd'hui en direction des classes populaires: pas assez de locuteurs, pas assez solvables. L'édition franco-centrée vivote. La bulle se fonde sur le statut du français, langue scolaire, administrative et politique, sur le renforcement extérieur par la francophonie et intérieur par le "Mali-France " - sur la force même du français du Mali, langue vernaculaire et africaine, riche, plastique capable de saisir le "paysage " local et international (dans l'acception la plus large d'une géographie vécue) par une langue précise, drôle, chaleureuse et nourricière pour le locuteur lettré de la langue française de France. La bulle "langues nationales " est de plus en plus centrée sur le bamakokan (la variante bamakoise du bamanankan), langue nationale véhiculaire émergente selon Morante (2009) et de nombreux acteurs locaux. De fait, le Mali perdrait régulièrement de la diversité linguistique, non au profit du français, mais à celui du bamakokan. L'idéologie de la diversité, la décentralisation, les fortes croissances des effectifs scolaires, le nationalisme linguistique favorisent les partisans de la relocalisation linguistique et de l'usage officiel des langues nationales. Au-delà de ces débats maliens très fortement clivés, la politique dite du curriculum (une scolarité complète en langues nationales avec le français en langue seconde croissante), qui remplace l'expérimentation de la pédagogie convergente $(\mathrm{PC})$, représente une opportunité socialement acceptable de baisse des coûts et de hausse de l'efficacité scolaire, un contournement possible du mur jusqu'à présent infranchissable de I'alphabétisation universelle au Mali. La bulle arabophone, très minoritaire, semble dynamique après plusieurs mouvements de réforme, à la fin de la période coloniale, dans les années 1970 et à nouveau aujourd'hui : après une véritable révolution logistique et intellectuelle, les médersas reconnues par l'État semblent intégrées à la société malienne contemporaine. La stagflation de la littéracie naît pour une part du faible chevauchement de ces trois bulles, de leur faible synergie. La fragmentation du marché national, la très inégale allocation des ressources, la spéciation stricte des formes des deux principales bulles linguistiques (écrit en français, oral en langues nationales) empêchent le marché, non seulement de croître, mais d'abord de se constituer, alors même que la population croît, elle, très fortement. Cette stagflation empêche également l'unification de l'espace politique formel (registres des partis politiques, registres du droit) lieu habituel des débats culturels et éducatifs, créant ici une boucle de rétro-action négative singulière: le débat officiel sur les langues nationales se fait principalement en français, dans une catégorie numériquement réduite de la population concernée. Les dynamiques linguistiques depuis I'Indépendance semblent ainsi contrôler une partie importante des données de la littéracie malienne, de l'échec de l'utopie scolaire en " langue étrangement nationale " (le français) à la relocalisation et à la polarisation en " langue nationale émergente » (le bamakokan et autres langues). La capitale et les élites sont les deux ensembles sociaux-spatiaux qui possèdent le moins d'écoles à curriculum et résistent ainsi à l'action publique de relocalisation linguistique. Ce clivage linguistique détermine des positions et des opinions très tranchées.

\section{Le transcolonialisme dans la littéracie ou le « Mali-France »}

Le chercheur français qui travaille dans un territoire, une société anciennement colonisés par la France ne peut faire l'économie d'une double précaution, ne pas 
prendre l'ambiance francophone dans laquelle il va le plus souvent être baigné pour le seul pays réel, ne pas prendre son modèle culturel de référence pour un objet d'exportation indiscutable. Ces précautions peuvent s'appliquer aussi au modèle de la Françafrique (Verschave, 1998) pourtant fortement critique des pratiques mafieuses des élites politico-économiques françaises et africaines. II n'est pas lieu de discuter cette analyse ici, mais de rappeler que l'épaisseur et la diversité des sociétés africaines ne peuvent s'en suffire, car c'est la société toute entière qui " joue " transcolonial. Cette interaction nous semble dessiner un "Mali-France " bien plus complexe que prévu, une réalité et une représentation opérant tant en France sur l'image du Mali francophone et de la présence malienne en France, qu'au Mali même sur l'image de la France, de la langue française, du groupe malien francophone et des positions qui s'y rattachent. La relation spatialisée entre les deux sociétés, malienne et française, se joue largement sur la langue. Ainsi, le "Forum national sur l'éducation » de 2008, forum participatif monté par les services du Premier ministre pour (tenter de) dénouer la crise du système éducatif malien a-t-il été une occasion d'une réitération du débat sur la politique linguistique du Mali. Dans cette opposition parfois étrange pour le tiers extérieur, entre "nationalistes vernaculaires" et "mondialistes francophones " (à moins qu'il ne s'agisse du contraire - que les uns et les autres pardonnent au chercheur ces approximations langagières qui traduisent mal la complexité des positions respectives), nous retenons que les expatriés maliens diplômés, de cette vaste diaspora dispersée à travers le monde, reliés au pays, mais également entre eux grâce à la révolution de l'internet, ont su montrer au Mali même la diversité des politiques linguistiques à travers le monde et dépasser la seule expérience transcoloniale francomalienne. Ainsi, la sortie du "Mali-France " ne se réduit nullement à un enfermement nationaliste, isolationniste ou xénophobe, ni à une "malianisation", voire à une "mandinguisation " autoritaire de la société. Sortir du "Mali-France ", c'est au contraire agrandir, aux nouvelles échelles de la mondialisation, la connaissance et la réflexion maliennes sur l'expérience historique des politiques linguistiques dans le monde. C'est par une évasion scalaire, en somme, que le Mali peut s'échapper de l'enfermement transcolonial: diversité interne, système linguistique transfrontalier régional, politique africaine des langues, expériences asiatique et américaine du multilinguisme, sans oublier la construction d'un modèle de littéracie nationale développée et intégrée, encadrée par l'action publique souveraine. Nous verrons plus loin que la constitution d'un marché de la littéracie plus " juste " (c'est-à-dire plus proche des potentialités de la société locale) peut être considéré comme un objectif de développement, mais aussi, et plus sûrement encore, comme un objectif raisonnable d'un nationalisme malien bien compris (en revisitant radicalement le legs et la mythologie de la Première République). Le prendre enfin comme un objectif esthétique, culturel et économique pour les acteurs de la filière et les autres habitants a encore plus de sens. Nous parlons là des biens de haute nécessité et d'une forme de l'authenticité.

\section{Aménager la culture, petit essai d'éthique informelle}

Dans la perspective qui est la nôtre, celle d'une recherche-action, la géographie de la littéracie s'entend, au-delà des acteurs, comme une réflexion sur l'action, civile et publique, passée, présente et à venir. II s'agit de penser l'aménagement du territoire étendu à la culture, ou mieux, de penser ensemble le " ménagement » du territoire et 
de la culture. Dans le cas malien, il faut s'affranchir d'un certain nombre de mythologies agissantes et de positions solidement établies pour penser l'autonomie d'un secteur émergent. Proposer aux acteurs une réflexion sur des outils existants ou à construire prend ici la forme d'un atelier/compte-rendu d'enquête en février 2012 dans le programme de "la rentrée littéraire malienne", le festival endogène de littérature de Bamako. Nous exposons ci-dessous quelques éléments de ce retour d'enquête, sur la question de l'alphabétisation en langues nationales, sur la concurrence du livre français, enfin sur la diversité des outils qui rendent possible la formation d'un marché national de la lecture-écriture cohérent et l'accès à une littéracie universelle pour l'ensemble des citoyens maliens.

\section{Une utopie scolaire pragmatique, relocaliser en langues nationales}

Dans le Mali diglossique contemporain, une faille centrale français/langues nationales sépare les nombreux citoyens non lettrés non scolarisés non francophones du petit groupe lettré scolarisé en français. Il existe une troisième catégorie difficile à quantifier, les adultes alphabétisés en langues nationales, au travers de dispositifs très hétérogènes de néo-alphabétisation, autoproduits ou à l'initiative de l'État, ainsi qu'un groupe significatif de francophones non lettrés. En détournant Deschooling society (Illich, 1973), nous disons que dans les processus observables aujourd'hui au Mali, il ne s'agit pas tant de "déscolariserla société " (qui ne l'est pas assez), que de " déscolariser la littéracie " (deschooling literacy) qui l'est trop, tout en " rescolarisant l'école ", c'est-à-dire en la recentrant sur des objectifs scolaires consistants et raisonnables - former de manière efficace l'ensemble d'une classe d'âge dans les langues fonctionnelles de la société. La dynamique éducative malienne est aujourd'hui de plus en plus hétérogène et polarisée: écoles publiques $49 \%$, privées $8,5 \%$, communautaires $27 \%$ et médersas 15,5\% (2010). Cet éclatement est né du recul des scolarités pendant toute la décennie 1980, période de crise caractérisée par un appauvrissement intellectuel et matériel des écoles fondamentales pendant ce qu'il faut appeler "l'ajustement structurel de la littéracie ». La reprise de la scolarisation lors de la décennie suivante se fait aux nouvelles conditions et selon l'idéologie des bailleurs internationaux, lesquelles favorisent la fragmentation et la privatisation du système éducatif, dont l'État malien a aujourd'hui partiellement perdu le contrôle. Parfois totalement délégitimée aux yeux des habitants (baisse du niveau, hausse des coûts, baisse des opportunités de placement socio-professionnel, corruption, harcèlement sexuel) et toujours très inégalitaire (espace, classe, genre), l'école malienne apparaît à la fois empêtrée dans une crise majeure et travaillée par une dynamique de résilience de celle-ci. À une échelle régionale, ouest-africaine, la relocalisation linguistique de l'école est un objectif majeur. Parmi les racines à retrouver pour refonder la gouvernance en Afrique, il est évident que les langues autochtones les plus parlées ou comprises ont un rôle fondamental et irremplaçable à jouer. En effet, dans les anciennes colonies françaises, l'enseignement est dispensé exclusivement en français soit dès la maternelle ou le cours préparatoire, soit à partir du CE2. Tel quel, il engendre de graves effets pervers : (...) l. parce que [l'enfant] entre dans le cycle primaire avec " un retard de six années de compétence linguistique " (cf. Agbidinoukoun, Dr en sciences du langage) très difficile à rattraper. Le jeune élève comprend donc moins vite, souvent mal ou même pas du tout. Aussi, les taux de redoublement et d'abandon sont-ils très élevés. Le pourcentage d'enfants scolarisés 
terminant le cycle primaire reste faible et le nombre de ceux qui accèdent à l'enseignement secondaire, dérisoire par rapport à l'ensemble de la population. 2 : Un temps et des efforts démesurés sont imposés aux enfants pour assimiler et maîtriser la langue française au détriment de l'acquisition des connaissances scientifiques et techniques dont ils auraient besoin pour se développer ${ }^{6}$. Les études maliennes sur le bénéfice scolaire des écoles expérimentales créées dans les années 1980 et 1990 attestent que les enfants éduqués en langue maternelle progressent plus vite et sont plus performants scolairement que ceux qui sont éduqués en "langue étrangement nationale " : il y a un coût cognitif à l'apprentissage du français, une langue étrangère, comme langue de travail scolaire, dans le cadre d'un apprentissage souvent réalisé de manière répétitive et par cœur dans des classes à effectif nombreux. Les termes du débat sont parfois violents, car, pour les nombreux et visibles partisans du français, ne pas scolariser en français, ce n'est bon ni pour la carrière scolaire et sociale de l'enfant, ni pour l'accès de la société malienne à la culture scientifique mondiale, mais, pour d'autres, ne pas scolariser en langues nationales et ne pas faire advenir celles-ci au monde, c'est se nier et nier la richesse humaine, locale et nationale, individuelle et collective et empêcher le Mali d'être au monde, souverain. À charge alors pour le système scolaire national de développer le français et l'anglais comme langues secondes.

Document 1 : compétence linguistique dans l'une des langues nationales déclarée par les maîtres des écoles à pédagogie convergente, année 2004

\begin{tabular}{|l|l|l|}
\hline langues & enseignants & $\%$ \\
\hline 1- Bamanankan & 18180 & 72,14 \\
\hline 2- Songhay & 2119 & 8,4 \\
\hline 3- Dogoso (dogon) & 1057 & 4,19 \\
\hline 4- Fulfulde (peulh) & 612 & 2,42 \\
\hline 5- Syenara (sénoufo- & 388 & 1,53 \\
\hline a) & & 1,56 \\
\hline $\begin{array}{l}\text { 6- Mamara (sénoufo- } \\
\text { b) }\end{array}$ & 414 & 1,64 \\
\hline 7- Bomu (bobo) & 636 & 1,63 \\
\hline 8- Soninké & 412 & 1,33 \\
\hline 9- Tamasheq & 337 & 0,36 \\
\hline 10- Bozo & 91 & 1,72 \\
\hline 11- Khassonké & 435 & 2,04 \\
\hline Indéterminés & 516 & \\
\hline
\end{tabular}

Source : rapport AUF, la place du français dans enseignement au Mali ${ }^{7}, 2006$, page 26

6 Louise-Marie Diop-Maes (Mme Cheikh Anta Diop), Docteur d'Etat en géographie humaine, Stratégie pour l'utilisation dans l'enseignement et dans l'administration des principales langues parlées ou comprises par les habitants, instauration d'un système éducatif rationnel et fonctionnel http://www.institut-gouvernance.org/publications/publication1/bamako-243.html

7 http://www.dlf.auf.org/IMG/pdf/Redaction_def-pdf1.pdf 
Dans cette relocalisation linguistique, on observe la possibilité d'une langue nationale véhiculaire, qui réduit sensiblement la représentation babéliste du Mali sans contraindre à l'uniformisation linguistique autoritaire.

\section{Le livre français au Mali, régler la concurrence déloyale}

Le caractère déloyal d'une pratique peut se mesurer. Objectiver la concurrence du livre français sur le marché malien, c'est la mesurer en comparant les deux filières : du côté français, un très ancien artisanat intellectuel devenu depuis plus d'un siècle une industrie de consommation intégrée, aujourd'hui transnationale et oligopolistique, fondée sur une idéologie universaliste incarnée notamment par la francophonie, du côté malien, un ancien artisanat arabophone qui a évolué vers une très petite industrie, récente, modeste, parcellisée dépourvue de filière complète et d'accès à la totalité de son marché, enfermée dans la langue française et les marchés publics. Il est difficile de soutenir que les acteurs maliens du livre sont en situation de se mesurer loyalement à leurs concurrents français. Quant à dire le caractère subjectif de la pratique, c'est décrire l'état de conscience des acteurs des deux filières. Croire comme le fait le Syndicat national de l'édition (syndicat patronal des grands éditeurs français) dans son rapport sur La présence du livre français dans les pays francophones du sud, memorandum au SNE (Cousin, 2006) que tout va bien et que les éditeurs français agissent comme de grands bienfaiteurs de l'Afrique relève d'une autre ère géopolitique. Dans la même logique, les dons de livres d'origine française (mais aussi belge, suisse ou québecoise) affectent fortement le marché malien. Cette concurrence déloyale ne concerne pas seulement la partie malienne. En effet, seuls les gros éditeurs français vendent au Mali, compte-tenu de la complexité de la commande et du transport des livres entre les deux pays, affectant la perception malienne de l'état de l'édition en France et les tenant à l'écart d'un grand nombre d'innovations et de pratiques alternatives au mainstream éditorial français. Dans le sens contraire, exception faite des éditions du Figuier, la filière malienne n'exporte que des manuscrits et des auteurs et importe des produits finis neufs ou de seconde main. Nous retrouvons dans cet échange culturel bien des aspects de l'échange inégal Nord/Sud tel qu'il a été décrit depuis les années 60. Que peut-on alors faire devant un tel schéma? Interdire ou surtaxer le livre français entrant au Mali rappelle des précédents fâcheux et donnerait des signaux politiques particulièrement contraires au développement d'un environnement favorable à la littéracie. Le partenariat francomalien questionne plutôt le "travailler ensemble" et l'obligation pour la partie française d'accepter l'idée que la filière malienne du livre a le droit de vivre et de se développer. 
Document 2 : régler la concurrence du livre français au Mali, quelques hypothèses de type gagnant-gagnant

\begin{tabular}{|c|c|c|}
\hline Filière française & Proposition & Filière malienne \\
\hline Oligopole & $\begin{array}{l}\text { Un mécanisme d'aide publique franco- } \\
\text { malien lié aux gros éditeurs: } \\
\text { l'État français (et les éditeurs } \\
\text { volontairement) finance la baisse du } \\
\text { prix de vente des livres français au Mali, } \\
\text { tandis que l'État malien taxe à l'entrée } \\
\text { au Mali les livres d'un montant } \\
\text { équivalent à la baisse subventionnée } \\
\text { (ou soutenue) du prix }\end{array}$ & $\begin{array}{l}\text { Le produit de la taxe malienne } \\
\text { (subventionnée par l'APD française } \\
\text { et les gros éditeurs) abonde le } \\
\text { Centre National de la Littéracie du } \\
\text { Mali, en charge de l'innovation et } \\
\text { de la pérennisation de la filière } \\
\text { malienne (à créer) } \\
\text { risques : blocages politiques en } \\
\text { France et bureaucratie/corruption } \\
\text { au Mali }\end{array}$ \\
\hline $\begin{array}{l}\text { Franges } \\
\text { créatives }\end{array}$ & $\begin{array}{l}\text { Partenariats non-étatiques : } \\
\text { multiplications des co-éditions du type } \\
\text { Alliance internationale des éditeurs, } \\
\text { avec soutien de fondations, échanges } \\
\text { de savoirs, de dratiques, de personnels } \\
\text { (workshops internationaux, stages et } \\
\text { projets partagés) } \\
\text { en cours } \\
\text { (Donniya, Jamana, Le Figuier) }\end{array}$ & $\begin{array}{l}\text { Ces co-éditions sont bénéficiaires } \\
\text { par forte réduction des coûts } \\
\text { unitaires de production } \\
\text { Bénéficiaires intellectuellement } \\
\text { par échange et émulation autour } \\
\text { des « bonnes pratiques " et trucs } \\
\text { du métier } \\
\text { risques : non réalisation par } \\
\text { manque de consistance des acteurs } \\
\text { et réduction des échanges culturels } \\
\text { liée au sécuritaire }\end{array}$ \\
\hline Rebut & $\begin{array}{l}\text { Formation des acteurs privés et } \\
\text { obligation de pérennisation liée au don } \\
\text { ponctuel de livres d'occasion : } \\
\text { diffusion des normes auprès des } \\
\text { donateurs et récipiendaires (démarche } \\
\text { qualité) } \\
\text { cofinancement obligatoire d'un lieu de } \\
\text { diffusion culturel économiquement } \\
\text { viable (soutien privé/public) } \\
\text { en cours } \\
\text { (spontanément ou par des structures } \\
\text { type BSF) }\end{array}$ & $\begin{array}{l}\text { Un stock doté d'une valeur } \\
\text { culturelle et économique certaine } \\
\text { permet de valoriser un lieu, un } \\
\text { poste de travail, mais seule la } \\
\text { pérennisation engage } \\
\text { positivement le développement de } \\
\text { la filière; } \\
\text { à lier, par exemple, aux } \\
\text { bibliothèques publiques ou aux } \\
\text { cybercentres, au multimédia (net, } \\
\text { téléphone), à un établissement de } \\
\text { formation, à une librairie rurale } \\
\text { risque : non réalisation par } \\
\text { manque de consistance des acteurs } \\
\text { et réduction des échanges culturels } \\
\text { liée au sécuritaire }\end{array}$ \\
\hline
\end{tabular}


Dans les trois registres de proposition, nous observons que des actions significatives ont déjà été engagées dans les franges créatives et le rebut par les acteurs eux-mêmes et sans aide publique significative. Ces astucieuses coopérations d'acteurs à faibles ressources financières peuvent être amplifiées sans les limiter au registre de la librairie formelle ou de la francophonie officielle. C'est l'innovation mutuelle et non la diffusion d'un modèle français stéréotypé difficilement généralisable qui importe. Concernant le cœur de cet échange inégal, les baisses de prix déjà ponctuellement travaillées entre grands éditeurs et État français ne convainquent pas, car c'est bien de la protection du marché malien dont il s'agit et non simplement de l'accès à des livres moins chers. Une partie significative de l'élite française étant attachée à l'exception culturelle et à la protection de ses propres outils culturels, de nombreux acteurs français sont en mesure d'admettre que toute exportation de livres français au Mali puisse financer un outil d'aide publique malien au livre (en fait à la littéracie) comparable au $\mathrm{CNL}^{8}$ français - sans limiter aucunement l'accès du public malien au livre français et notamment aux genres que l'édition malienne ne sera pas en mesure de produire avant un certain temps ou peut-être jamais, compte-tenu de l'effet de taille. En prenant en compte le chiffre difficilement vérifiable de 250000 livres français entrant au Mali annuellement, on voit qu'une baisse de $4 €$ par livre (partagée à $50 \%$ entre l'État français et les grands éditeurs concernés) permettrait à une taxe partenariale de dégager une somme théorique d'un million d'euros pour abonder un CNL malien ${ }^{9}$.

\section{Détours en littéracie, conditions pour une société civile cohérente}

La Bibliothèque Nationale du Mali a été créée en 1984. Lancé officiellement en 1986, le dépôt légal ne connaît guère de résultats probants jusqu'à sa relance active au milieu des années 2000. Cela d'autant plus que jusqu'en 2006 les livres maliens sont soit dépourvus de numéros ISBN, soit immatriculés avec un ISBN français obtenu par les éditeurs maliens auprès de l'AFNIL, l'organisme de gestion de I'ISBN français. La France impose I'ISBN à sa filière par un décret de décembre 1981 pris pour l'application de la loi relative au prix unique du livre. Le Mali créée son agence ISBN en 2006 (I'immatriculation ISBN est réclamée par les bailleurs). On comprend la forte symbolique de cette temporalité décalée, qui voit "l'indépendance ISBN » malienne être différée de 25 ans et une partie significative du "livre malien » (Donnya, Le Figuier, Jamana) avoir été enregistrée comme « livre français " pendant cette période (1986-2006). Aujourd'hui, et depuis 2006, l'AFNIL n'accepte plus les éditeurs maliens et, peu à peu, le dépôt légal sous ISBN malien progresse. De plus, une part significative

8 Le Centre National des lettres français / http://www.centrenationaldulivre.fr/

Autodéfinition sur le site du CNL: Établissement public du Ministère de la Culture et de la Communication, le Centre national du livre a pour mission d'encourager la création et la diffusion d'ouvrages de qualité, à travers divers dispositifs de soutien aux acteurs de la chaîne du livre (auteurs, éditeurs, libraires, bibliothèques, organisateurs de manifestations littéraires). Il est également un lieu de rencontres, d'échanges et d'actions interprofessionnelles. Cette caractéristique lui confère une place particulière et originale dans l'organisation administrative: éditeurs, auteurs et traducteurs, bibliothécaires et libraires sont étroitement associés aux actions mises en œuvre par le CNL.

9 Cette ressource ainsi envisagée sous la forme d'une fiscalité solidaire peut paraître improbable, son montant très exagéré, voire baroque. L'idée est pourtant bien d'affirmer qu'un CNL malien est non seulement possible, mais souhaitable pour l'émergence d'une filière nationale du livre à la hauteur de son marché potentiel, qu'il contribuera à réaliser, la société malienne alphabétisée. 
de l'édition malienne (brochures, livrets hors édition formelle) notamment en langues nationales échappe à tout enregistrement. Selon son inventaire actuel, la BNM contient 16472 ouvrages analogiques (13000 livres, 3400 thèses/mémoires et 72 titres de périodiques) contre 7500 à l'inventaire de 2003. C'est à peu près le nombre d'ouvrages d'une bibliothèque publique d'une petite ville française (10000 habitants). Nous observons également que le nombre de détenteurs maliens d'un ISBN ne dépasse pas 50 et celui des ISSN 63 (2010). Ici, revient le double effet de taille du Mali, une population modeste et parmi les plus jeunes du monde $(14,5$ millions d'habitants dont la moitié a moins de 15 ans), un lectorat lettré et francophone solvable très réduit (un taux global d'alphabétisation des adultes estimé à $26 \%$ sur la période 2005 2008, une réduction de cet effectif théorique en fonction de la langue et du faible pouvoir d'achat) qui contraignent un petit secteur professionnel incomplet et une production réduite, essentiellement en langue française.

L'action publique sensible à la question des biens de haute nécessité peut s'appuyer sur les acteurs locaux, sur la Constitution malienne de 1992 (articles 17, 18, 25 et 70 notamment), en particulier parce que celle-ci offre un cadre évolutif : le français est certes la langue d'expression officielle mais la loi fixe les modalités de promotion et d'officialisation des langues nationales (article 25). Elle peut aussi s'adosser à un mouvement transnational en faveur de la bibliodiversité et de la souveraineté culturelle. 


\section{Document 3 : figuration systémique d'une action publique et civile cohérente de développement de la littéracie nationale du Mali}

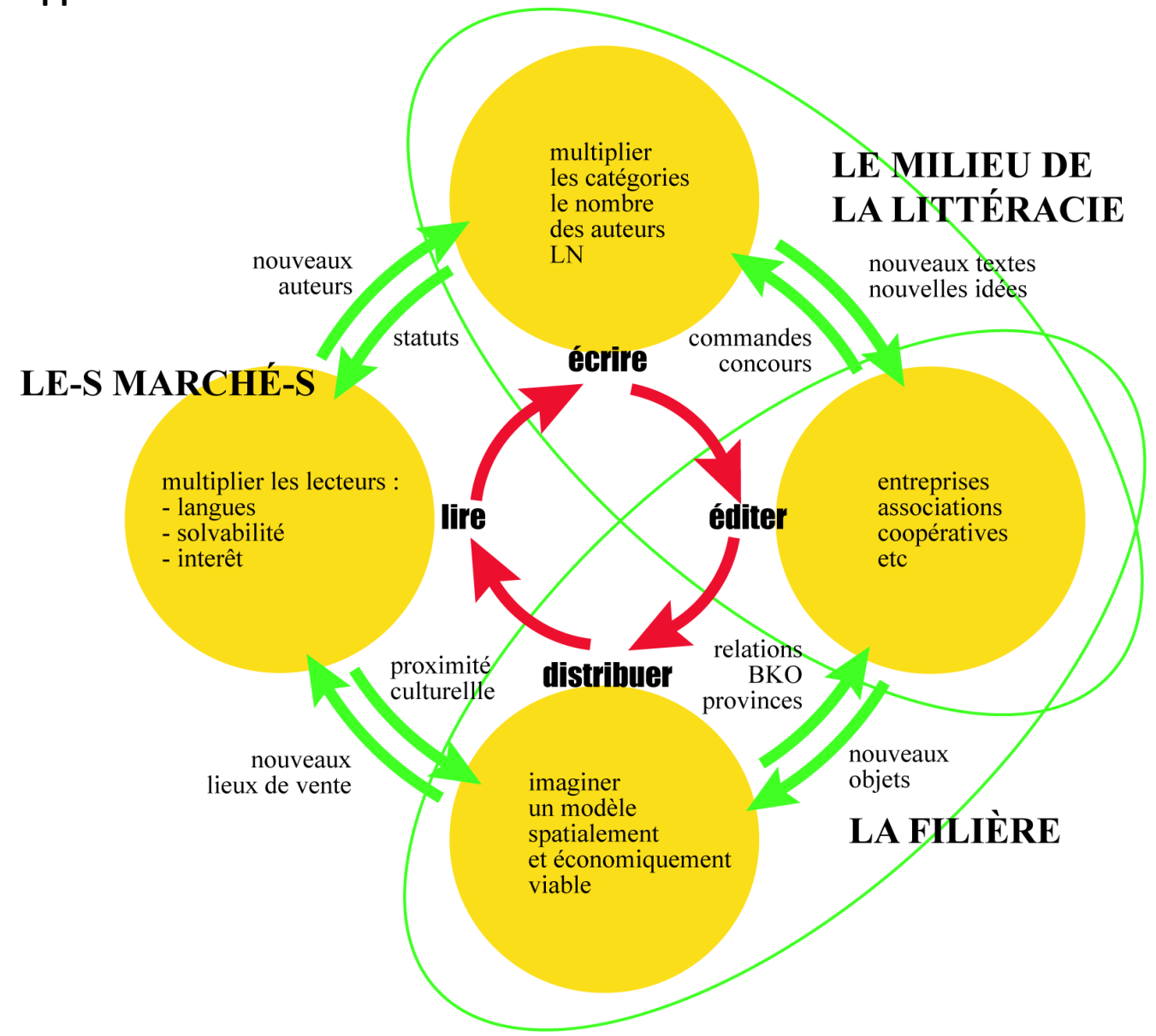

Source : inédit, Frédéric Barbe, infographie, Andrée Dubois, IGARUN, Université de Nantes.

Pour conclure, tenter ici un peu de géographie appliquée, "d'aménagement " ou plutôt de "ménagement " culturel, c'est bien de tenter de comprendre le débat interne à la société malienne, sans misérabilisme, ni contresens, en reconnaissant, parfois contre l'avis des acteurs dominants de la filière, de la valeur à tous les acteurs formels et informels, aux différentes bulles linguistiques, à toutes les formes culturelles, pour inventer sans peur de la trouvaille, du recyclage ${ }^{10}$, du bricolage, du marronnage - l'art de dégager de la liberté dans les espaces contraints - un nouveau modèle économique d'une littéracie de pays pauvre. La pauvreté économique ne peut signifier l'empêchement de la lecture-écriture et seules des idéologies inégalitaires peuvent soutenir aujourd'hui que les pauvres n'ont pas (besoin) de littéracie. Quel que soit le niveau de ressources d'un pays, l'action publique souveraine et celle des acteurs

10 La littérature de cordel brésilienne, moment historique de la littéracie populaire brésilienne émergente $\left(19^{\text {ème }} / 20^{\text {ème }}\right)$, aujourd'hui forme mutante en cours de patrimonialisation qui s'est déplacée pu sertão nordestin vers les villes littorales, peut représenter l'archétype de ces formes populaires combinant l'émergence du couple auteur/lecteur et de circuits de production/distribution simples, autofinancés et auto-gérés, pour servir une population en cours d'alphabétisation: une littérature de colportage qui rejoint sur certains points la littérature alternative la plus contemporaine des vieux pays alphabétisés, les franges de l'oligopole.. 
civils ont été et sont toujours toutes deux nécessaires pour créer et entretenir une filière nationale, nourricière de la société, vers tous les publics, intégrée à différentes échelles dans la littéracie mondiale. "L'ajustement structurel de la littéracie ", moment historique du consensus de Washington, a montré que la géographie de la culture ne peut se réduire à un " pur » marché, à fortiori dans un pays marqué par la figure transcoloniale.

\section{Bibliographie}

Barbe F. (2011) « Une formes souterraine et pro-active de littéracie, la spatialisation des bibliothèques privées ", séminaire Livre : création, culture et société, Université Paris Ouest Nanterre La Défense.

Barbe F. (2011) "Tentative d'épuisement du et des Prix Nobel de littérature", séminaire Livre : création, culture et société, Université Paris Ouest Nanterre La Défense.

Barbe F. (à paraître en revue) « La montée des îles Aran dans la bibliothèque mondiale, une glocalisation littéraire ".

Barber K. (2006) (ed) Africa's hidden histories : everyday literacy and making the self, Bloomington, Indiana University Press, $451 \mathrm{p}$.

Bayard P. (2007) Comment parler des livres que l'on n'a pas lus ?, Paris, Éditions de Minuit, 162 p.

Bayard P. (2009) Le Plagiat par anticipation, Paris, Éditions de Minuit, 154 p.

Becker H. $(1982,1988)$ Les Mondes de l'art, Paris, Flammarion, $379 \mathrm{p}$

Casanova P $(1999,2008)$ La République mondiale des Lettres, Paris, Éditions du Seuil, $504 \mathrm{p}$.

"Paris, Capitale littéraire " (2002) in Charle C. et Roche D. (2002) Capitales culturelles, capitales symboliques. Paris et les expériences européennes (XVIII'ème -XIXème siècle), Paris, Publications de la Sorbonne.

Chartier R. (1993, 2003) dir. Pratiques de la lecture, Paris, Éditions Payot, 323 p.

Chartier R. (1997, 2001) dir avec Cavallo G. Histoire de la lecture dans le monde occidental, Paris, Éditions du Seuil, 587 p.

Chartier R. (1997) Le Livre en révolutions, entretiens avec Jean Lebrun, Paris, Éditions Textuel, $159 \mathrm{p}$.

Coquery-Vidrovitch C. (1992) L'Afrique occidentale au temps des Français, colonisateurs et colonisateurs, c. 1860-1960, Paris, Éditions La Découverte, 460 p.

Cousin B. (2006) La présence du livre français dans les pays francophones du sud, memorandum au Syndicat nationale de l'édition, Paris, SNE, en ligne sur le site du SNE, 33 p.

Dard F. (2000) "Entretien avec Jérôme Garcin » Le Nouvel Observateur, numéro 2000/1858.

Doquet A (2009) « Le terrain des notes. Enquête, notes de terrain et raisonnement de l'anthropologue ", Langage et Société, 2009/127.

Fabre D. (1993) Écritures ordinaires, Paris, Éditions P.O.L, 374 p.

Goody J. (1977, trad 1979) La Raison graphique. La domestication de la pensée sauvage, Paris, Éditions de Minuit, 275 p.

Goody J. (1993, trad 1994) Entre l'oralité et l'écriture, Paris, PUF, 323 p. 
Guaino H. (2007) Discours de Dakar, prononcé par Sarkozy N. à l'Université CheikhAnta-Diop de Dakar.

Hébrard J., Chartier A-M. (2000) Discours sur la lecture (1880 - 2000), Éditions Arthème Fayard - BPI, centre Georges Pompidou, 762 p.

Hébrard J., ChartierA-M., Clesse C. (1998) Lire-Écrire : entrer dans le monde de l'écrit, Éditions Hatier, $191 \mathrm{p}$.

Hoggart R.(1957, 1970) La culture du pauvre, Paris, Éditions de Minuit, 420 p.

Illich I. (1971, 1997), Une société sans école, Paris, Éditions du Seuil, 219 p.

Lahire B. $(1999,2005)$ L'invention de l'illettrisme. Rhétorique publique, éthique et stigmates, Paris, Éditions La Découverte, $370 \mathrm{p}$.

Lahire B. (2006) La Condition littéraire: la double vie des écrivains, Paris, Éditions La Découverte, $619 \mathrm{p}$.

Le Potvin S. (2005) Lettres maliennes, figures et configurations de l'activité littéraire au Mali, Paris, Éditions L'Harmattan, 337 p.

Manifeste pour les produits de haute nécessité (2009), Martinique, Guadeloupe, Guyane, Réunion, multidiffusion, Éditions Galaade, Institut du Tout-Monde et en ligne, $12 \mathrm{p}$.

Mbembé A. (2010) Sortir de la grande nuit, essai sur l'Afrique décolonisée, Paris, Éditions La Découverte, $246 \mathrm{p}$.

Mbodj-Pouye A. (2007) Des cahiers au village, socialisations à l'écrit et pratiques d'écriture dans la région cotonnière du sud du Mali, thèse de doctorat de Sociologie et Anthropologie de l'Université Lumière-Lyon 2, en ligne, 715 p.

Mbodj-Pouye A. (2010) (dir. avec Fraenkel B.), numéro spécial « Un courant de recherche sur l'écrit : les Literacy Studies ", Langage et Société, 2010/133.

Morante D. (2009) Le champ gravitationnel linguistique, avec un essai d'application étatique - Mali, Paris, Éditions l'Harmattan, 546 p.

Mara M. (2010) L'État au Mali, Géniteur de la Nation [Facteur d'Intégration, Catalyseur d'expansion], Bamako, auto-édition, $376 \mathrm{p}$.

Perec G. (1974, 2000) Espèces d'espaces, Paris, Éditions Galilée, 185 p.

Rancière J. (2007) Politique de la littérature, Paris, Éditions Galilée, 231 p.

Rancière J. (1987, 2004, 2009) Le Maître ignorant: cinq leçons sur l'émancipation intellectuelle, Paris, Éditions Fayard, 233 p.

Saïd E. (1993, trad 2000) Culture et Impérialisme, Paris, Éditions Fayard - Le Monde diplomatique, $558 \mathrm{p}$.

Sapiro G. (2009) Translatio. Le marché de la traduction en France à l'heure de la mondialisation., Paris, Éditions du CNRS, $427 \mathrm{p}$.

Sapire G. (2009) Les contradictions de la globalisation éditoriale., Paris, Éditions Nouveau Monde, coll. "Culture/Médias », 401 p.

Sepúlveda L. (1989, trad 1992) Le Vieux qui lisait des romans d'amour, Paris, Éditions Métailié, $136 \mathrm{p}$.

Smith S. (2003) Négrologie : pourquoi l'Afrique meurt ?, Paris, Éditions Calmann-Lévy, $248 \mathrm{p}$.

Travelé M. (1913) Petit Dictionnaire Français-Bambara et Bambara-Français, Paris, Éditions Paul Geuthner, 281 p. 